\title{
Origins of inspiration
}

\section{A four-dimensional view of two twentieth-century icons.}

\author{
Einstein, Picasso: Space, Time, \\ and the Beauty that Causes Havoc \\ by Arthur I. Miller \\ Basic Books: 2001. 320 pp. $\$ 30$
}

\section{John Christie}

The opening decade of the twentieth-century continues to hold a profound attraction for intellectual and cultural historians. It is not simply that obvious and major changes in painting, literature and music occurred at this time - the advent of modernism in Western culture - but that these changes seem so dramatically abrupt and, moreover, in some senses related to one another. The bolder souls among us also wish to include the sciences within this movement, so that the years 1905-09 witnessed a triple revolution at least, consisting of the departures initiated in painting by Picasso, Braque and Matisse in Paris, in music by Schönberg in Vienna, and in physics by Einstein in Bern.

Arthur Miller draws on his own substantial researches on the history of physics in this period to focus on two figures, Pablo Picasso and Albert Einstein. And, ultimately, on two key works - Picasso's Les Demoiselles d'Avignon of 1907 and Einstein's 1905 epochmaking paper describing special relativity, "On the electrodynamics of moving bodies". Miller's account proposes a definite historical relationship between the work of his two creative heroes. He contends that both depended significantly on a preoccupation with geometry, and that this preoccupation had its basis in the work of the great French philosopher-mathematician Henri Poincaré. Miller is not implying that there was any communicative influence acting between Picasso and Einstein, but that their common preoccupations had an underlying common cause.

Miller provides fascinating and thorough accounts of the conception, gestation and delivery of the key works. He supplements them with two further types of material. Interested in personal creativity, he gives biographically based accounts of his protagonists. He also adds contextual detail on the cultural, philosophical and scientific environments that bore strongly on Picasso's and Einstein's developing ideas. So we are informed not only of the technical significance of etherdrift experiments and the Lorenz contraction, of primitivist and photographically derived aesthetics, but equally of the significance of the bohemian, hashish-devoted culture that existed in Paris's Montmartre, where Picasso spent much of this period, and of Einstein's and Picasso's love-lives. All this adds considerably to the book's readability and interest, and turns what could have been a rather dry and

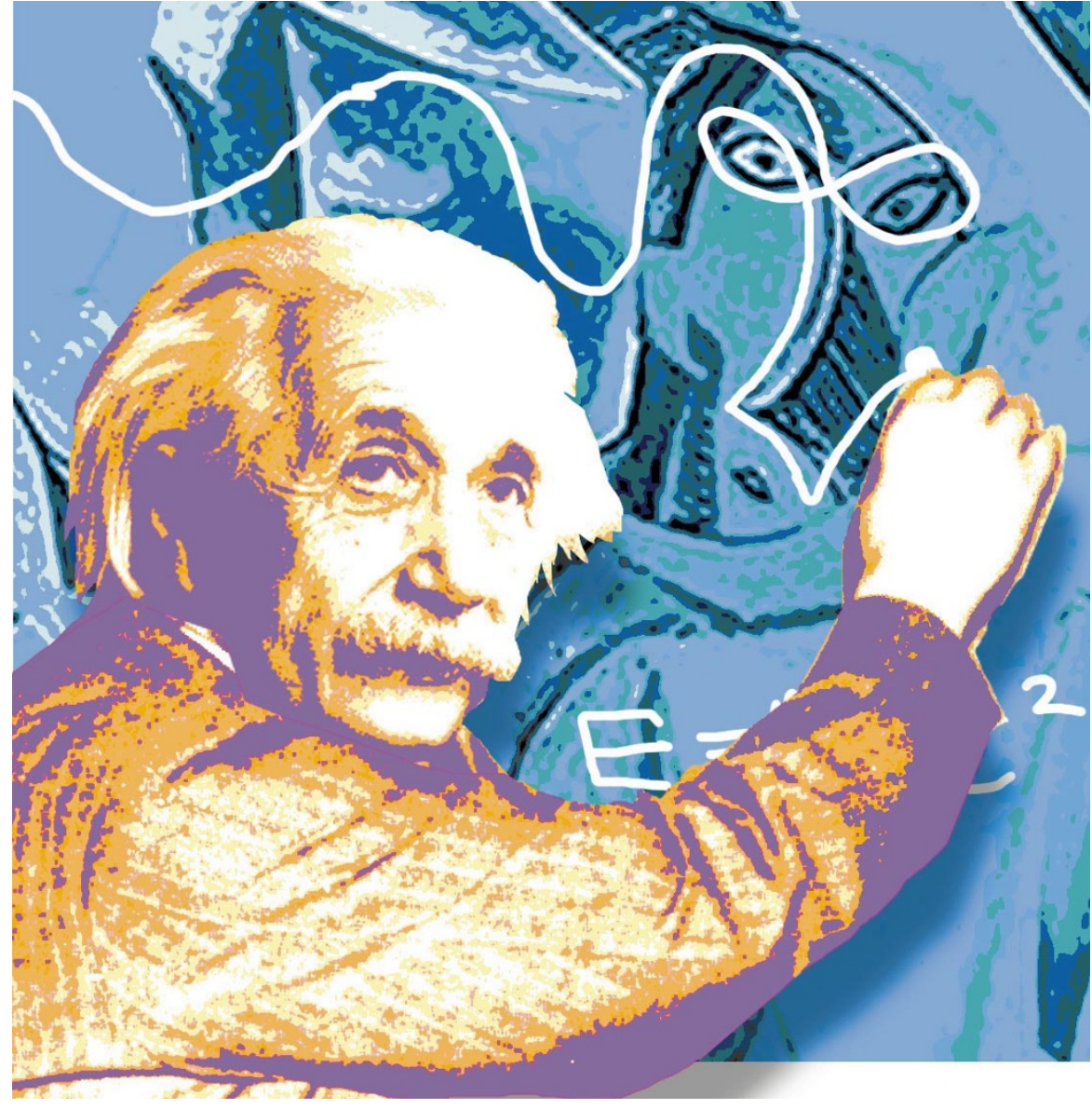

testing disquisition on geometrical technicalities into a lively and often compelling narrative. It captures what Miller clearly feels to be the sheer, exhilarating excitement of those times, places and events.

Both Einstein and Picasso are, of course, massively archived and researched figures, and Miller's analysis of their achievements makes substantial and commendable use of the most recent research on Picasso - particularly that of Anne Baldassari on Picasso's photography, and John Richardson's biography. But Miller adds something significant of his own about the geometric origins and development of Les Demoiselles. He identifies a plausible means by which the content of Poincaré's LaScience et l'Hypothèse could have reached Picasso, who in those years did not have a strong grasp of the French language. Maurice Princet, a mathematically learned actuary, was a member of Picasso's circle of intimates, la bande à Picasso. And, according to Miller, it was Princet who told Picasso about the non-euclidean and four-dimensional geometrical speculations of Poincaré and also of the French mathematician Esprit Joffre in a way that stimulated Picasso's ambitions to apply them to his painting.
This is a valuable nugget of historical research, and is generally persuasive. If one additionally buys into Miller's unabashedly Romantic, genius-led views of creativity and historical change, then his book offers an account of Picasso in Paris that is both new and convincing, with the added bonus of having Einstein in the same interpretive frame.

It is possible, nonetheless, to accept Miller's factual findings while remaining sceptical about his overall interpretation. When questioned, Picasso persistently and over a long period denied a geometric or scientific inspiration for Les Demoiselles. Miller's special pleading, that Picasso was not asked the question in the right way, is hardly convincing. It would be more to the point simply to insist that Les Demoiselles is a geometrical composition: the presentation of space and objects in the painting are well described as geometrical, despite Picasso's disavowal. But a larger difficulty concerns the kind of geometry involved. It is very difficult to maintain, with Miller, that a poincaresque 'four-dimensional' form is in play. Rather, three-dimensional depths, visually inaccessible in pictorial terms, are unfolded to the twodimensional flatness of the picture plane, 


\section{spring books}

giving the viewer the impression of seeing the picture from several perspectives, and thereby enhancing its visual effect. This is complemented by the fact that objects in the picture are geometrically broken up through the faceting that Picasso applied. Although it is interesting that contemporary occultists perceived four dimensions to the picture, this is difficult to validate from the point of view of the picture's composition. And it leaves open the question of how fundamental, or otherwise, is its derivation from Poincaré.

Perhaps surprisingly, the case for Einstein appears even more questionable. Although Poincarés place in the prehistory of special relativity is long acknowledged, there is the stumbling block that Einstein's Special Theory is not presented geometrically by Einstein. He treated electrodynamics not in any markedly geometrical way but rather by an abstract reconsideration of the relations between rigid rods, perfect clocks and light signals - that is, by proposing a theory of measurement that respecified the nature and meaning of fundamental physical quantities.

Miller's argument may be on stronger ground at a later period - with the formulation of analytical cubism by Picasso and Georges Braque, and with general relativity's fundamental space-time geometry. But for the main period on which the book focuses, 1905-07, his case, however vigorously and enjoyably portrayed, remains tantalizingly unmade.

John Christie is at the Division of History and Philosophy of Science, University of Leeds, Leeds LS2 9JT, UK.

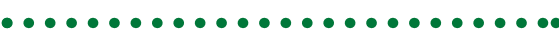

... but what does 'blue' smell like?

Synaesthesia: The Strangest Thing by John Harrison

Oxford University Press: 2001. 250 pp.

$\mathfrak{E} 16.99, \$ 35$

\section{Ilya Farber}

Of all the neurological disorders known to modern science, synaesthesia may be the only one that routinely inspires envy in those who study it experimentally. The experience of the synaesthetic seems somehow richer and more compelling; after all, who wouldn't want to experience music in full, glorious colour? Most 'normal' humans experience occasional cross-modal associations, perhaps with the assistance of Mahler or LSD, but this seems thin compared with the inner world of the synaesthetic painter who always experiences the spoken word 'Moscow' as "darkish grey, with spinach-green and pale blue in places". How can one not envy the woman who experiences shapes accompanying smells, so that the smell of wintergreen evokes "ragged

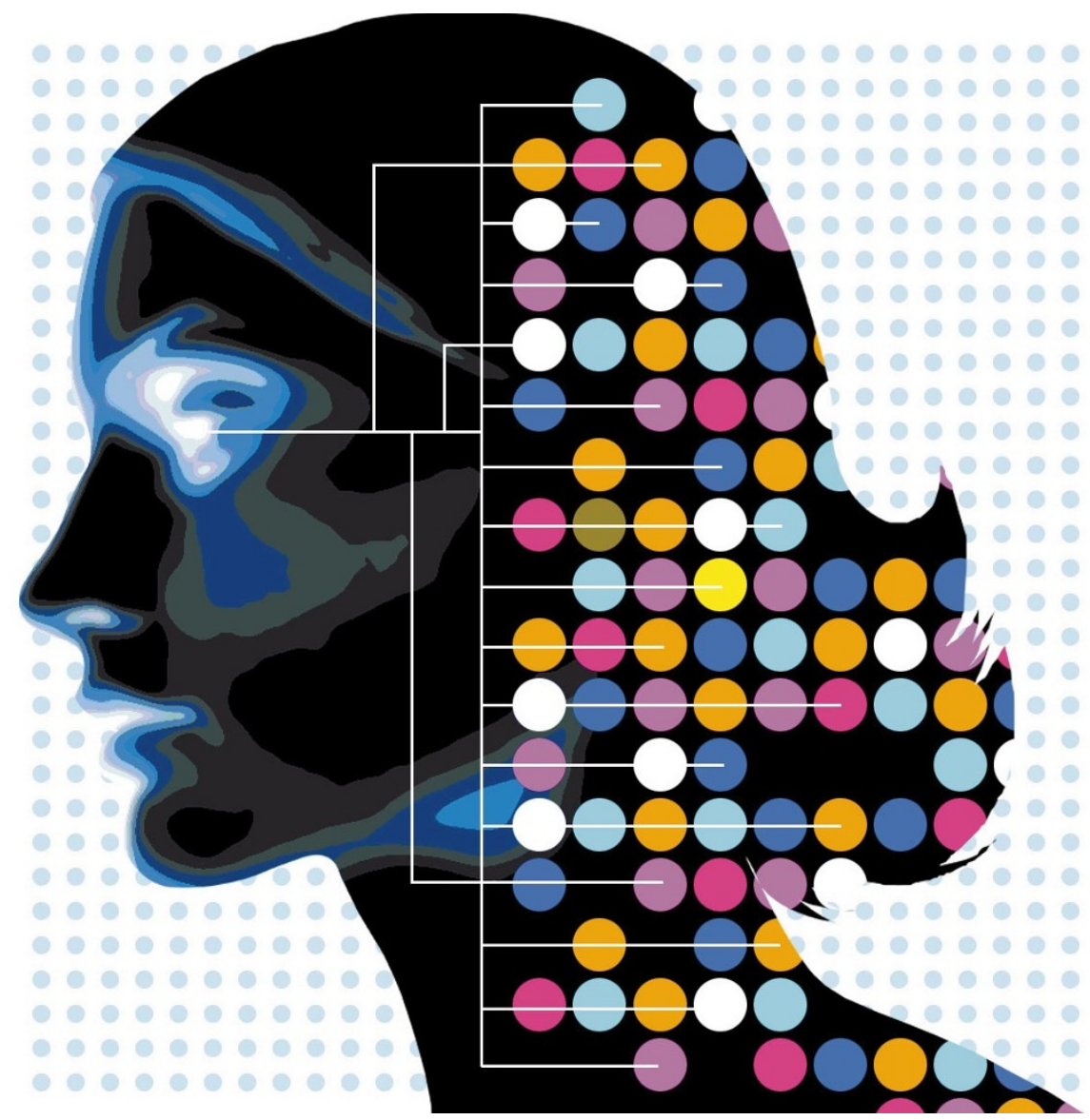

edges", and peanut butter smells like "things falling down and backward"?

For the past decade, John Harrison has been studying such people, and his book provides an accessible survey of what is known about this most intriguing of disorders. Although he wrote the book alone, most of the work he reports was done in collaboration with Simon Baron-Cohen, and the theory that they jointly developed forms the basis for much of Harrison's approach.

This theory begins with the well-established fact that all humans are born with substantial interconnections among the different sensory pathways in the brain. These normally die off within the first six months of life, but if a pathway's normal source of input is cut off, as occurs in congenital blindness, it may retain its connections to other pathways. Harrison draws the reasonable inference that in synaesthesia some of these pathways are somehow preserved into adulthood. $\mathrm{He}$ supports this hypothesis by pointing out that synaesthetics usually say they have had the condition for as long as they can remember, and that (according to an analysis of the limited pedigree data available) the most common form of the condition appears to be genetic - specifically, an X-linked dominant trait that is partially lethal in males.

Unfortunately, definitive tests for neuroanatomical connectivity are impossible in humans, as they require the use of injected tracers, followed immediately by postmortem sectioning and staining of the brain. Harrison does present neuroimaging data showing that, when colour-word synaesthetics listen to words while blindfolded, some of the brain areas activated are those that have been implicated in colour processing. But most of these areas are known to be driven by stimuli other than just colour even in normal people, and some of the areas activated in Harrison's imaging studies are not sensory at all. Even the clearest result, which shows activity in a highly specialized colour area, cannot identify the source of the activity. So it remains a possibility that the visual pathway is being driven not by cross-wiring but by something more like visual imagination.

Overall, Harrison's theoretical discussions are relatively superficial; where the book really shines is in discussing the data. Reading this book is like looking over a detective's shoulder at the collection of casefiles and newspaper clippings spread out on his desk. Harrison has done all the arduous work of gathering the raw data and sorting through historical reports; now he invites the reader to join him in sifting through it for patterns and meaning.

And a fascinating deskful it is. Harrison calculates that the overall population prevalence of synaesthesia is at least 1 in 2,000; most of the synaesthetics he has found are word-colour synaesthetics, and about 85\% of these are women - though, perhaps unsurprisingly, all of the famous historical candidates for synaesthesia that Harrison found are men.

It turns out that the colour of a word is usually determined by its first letter, and that 